\title{
2014 Eisenbud Prize for Mathematics and Physics
}

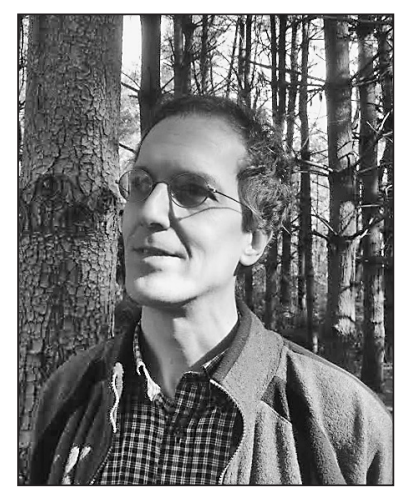

Gregory W. Moore
GREGORY W. MOORE was awarded the 2014 Leonard Eisenbud Prize for Mathematics and Physics at the 120th Annual Meeting of the AMS in Baltimore, Maryland, in January 2014.

\section{Citation}

The 2014 Leonard Eisenbud Prize for Mathematics and Physics is awarded to Gregory W. Moore for his group of works on the structure of fourdimensional supersymmetric theories with extended supersymmetry. His works on supersymmetric solitons in a variety of contexts-including black holes in supergravity, branes in string theory, and monopoles in gauge theory-have led to an explanation of the wall-crossing phenomena in the BPS spectrum. Moore's research has injected new physical ideas and created new constructions in the mathematical fields of cluster algebras, integrable systems, and hyperkähler geometry.

In particular the following papers are cited:

1. Frederik Denef and Gregory W. Moore, "Split states, entropy enigmas, holes and halos", Journal of High Energy Physics 129 (2011), no. 11, i, 152 pp.

2. Duiliu-Emanuel Diaconescu and Gregory W. Moore, "Crossing the wall: branes versus bundles", Advances in Theoretical Mathematical Physics 14 (2010), no. 6, 1621-1650.

3. Davide Gaiotto, Gregory W. Moore, and Andrew Neitzke, "Four-dimensional wall-crossing via three-dimensional field theory", Communications in Mathematical Physics 299 (2010), no. 1, 163-224.

4. Davide Gaiotto, Gregory W. Moore, and Andrew Neitzke, "Wall-crossing, Hitchin systems, and the WKB approximation," Advances in Mathematics 234 (2013), 239-403.

5. Davide Gaiotto, Gregory W. Moore, and Andrew Neitzke, "Spectral networks", Annales Henri Poincaré 14 (2013), no. 7, 1643-1731.

DOI: http://dx.doi.org/10.1090/noti1113

\section{Biographical Sketch}

Gregory W. Moore received his A.B. in physics from Princeton University in 1982 and his Ph.D. in physics from Harvard University in 1985. He then joined the Harvard Society of Fellows and in 1987 became a five-year member at the Institute for Advanced Study (IAS) in Princeton. In 1989 he joined the faculty at Yale University. He moved to the Department of Physics and Astronomy at Rutgers University in 2000. He has held visiting professorships at the Kalvi Institute for Theoretical Physics (KITP) in Santa Barbara, California, and at the IAS. The Inspire HEP database lists 170 papers coauthored by Professor Moore on physical mathematics, with an emphasis on geometrical structures in physics. Most notably he has worked on rational conformal field theories (with applications to condensed matter physics), two-dimensional quantum gravity and matrix models, topological field theories, string dualities and D-branes, applications of $K$-theory to string theory, connections between number theory and supersymmetric black holes, and the properties of BPS states of supersymmetric theories with an emphasis in recent years on their wall-crossing properties and relations to hyperkähler geometry. He is a member of the American Physical Society, the American Mathematical Society, the American Academy of Arts and Sciences, and a general member of the Aspen Center for Physics.

\section{Response from Gregory W. Moore}

I am deeply honored, and not a little surprised, to be the sole recipient of the 2014 Leonard Eisenbud Prize for Mathematics and Physics. First and foremost I would like to thank my collaborators, Frederik Denef, Emanuel Diaconescu, Davide Gaiotto, and Andrew Neitzke, for their essential insights and enthusiasm for what turned out to be a very fruitful line of enquiry. I was the senior author only in years-not infrequently it was my collaborators who were leading the charge.

Since the AMS has requested a response to this award, I will use the opportunity to sketch my 
viewpoint on how the work mentioned in the citation fits into a broader context and then to conclude even more broadly with some thoughts on the place of physical mathematics in the contemporary relation of the mathematical and physical sciences.

A central theme of the work in the citation is the behavior of four-dimensional theories with $N=2$ supersymmetry. The 1994 breakthrough of Nathan Seiberg and Edward Witten amply demonstrated that quantum field theories with extended supersymmetry constitute a Goldilocks class of theories which are special enough to admit exact nontrivial results on their dynamics but general enough to exhibit a host of nontrivial phenomena in quantum field theory. The promise of the Seiberg-Witten breakthrough is twofold: First, one can make exact statements about how the massless particles in the theory interact at low energies. Second, one can make exact statements about the spectrum of the Hamiltonian for a subsector of the Hilbert space of states called the "BPS subspace". One of the key features of these theories is that the vacuum state is not unique, but rather it is parameterized by a manifold (which carries a special Kähler metric). Thus, an example of the first kind of result is an exact description of the strength of Coulomb's law as a function of the vacuum parameters.

I would guesstimate that there have been well over ten thousand physicist years devoted to the intense investigation of four-dimensional $N=2$ field theories. Nevertheless, the full promise of the Seiberg-Witten breakthrough has not yet been fully realized. Regarding the first kind of result, important and nontrivial insights continue to be uncovered up to the present day in the works of Nikita Nekrasov, Samson Shatashvili, Vasily Pestun, Edward Witten, and a host of others revealing relations to integrable systems and many other things. The papers mentioned in the citation address the second kind of result: deepening our understanding of how to compute the so-called BPS spectrum for ever larger classes of $N=2$ theories. The key theme in these papers is that, as a function of vacuum parameters, the BPS spectrum can be discontinuous across real codimension 1 loci in the space of vacuum parameters. An important point is that there exists a very beautiful formula which expresses how this spectrum changes. Since a real codimension 1 locus is a wall, the formula is known as a wall-crossing formula. The history of this formula is far too complicated to be explained here, but I will note that it began with a formula of Sergio Cecotti and Cumrun Vafa for the decays of solitons in two-dimensional quantum field theories, and, in addition to my work done in collaboration with Denef, Diaconescu, Gaiotto, and Neitzke, essential insights and breakthroughs were made in the context of pure mathematicsand motivated by pure mathematics-by Maxim
Kontsevich and Yan Soibelman and separately by Dominic Joyce and Yinan Song in their work on generalized Donaldson-Thomas invariants for Calabi-Yau categories. Research into BPS states continues to be a very active subject.

As indicated in the citation, the investigations into the BPS spectrum have led to a wide variety of unexpected and rich connections to many branches of pure mathematics. Like a beautiful flower which continues to unfold and dazzle, the deeper the probe, the richer the emergent mathematics. In addition to the relations of four-dimensional $N=2$ theories to hyperkähler geometry, cluster algebras, cluster varieties, and integrable systems, several other remarkable links to subjects in pure mathematics have been discovered by many mathematicians and physicists in the past several years. The full list is too long to mention here, but some prominent examples include deep relations to geometric representation theory and nontrivial connections with modular tensor categories and two-dimensional conformal field theory.

In view of the extraordinary richness of the field, one might well wonder if there is some simplifying and unifying viewpoint on all the above connections. Indeed, the following is widely believed by many mathematicians and physicists: A striking prediction of string theory from the mid-1990s (in the hands of Edward Witten, Andrew Strominger, and Nathan Seiberg) is that there is a class of six-dimensional interacting conformal quantum field theories known as the $(2,0)$-theories. Many of the beautiful connections alluded to above can be traced to the very existence of these theories. On the other hand, these six-dimensional theories have not yet been fully formulated in any systematic way. There is no analog of a statement for nonabelian gauge theory, such as "Make sense of the path integral over connections on a principal bundle weighted by the Yang-Mills action." Indeed the very mention of the $(2,0)$-theories is greeted by some scientists with an indulgent smile. But many of us take them seriously. An important problem for the future is a deeper understanding and formulation of these theories.

For reviews giving a more extensive explanation of these matters, the reader could consult my review talk at Strings2011 in Uppsala, Sweden, my review talk at the 2012 International Congress on Mathematics and Physics in Aalborg, Denmark, or my 2012 Felix Klein lectures delivered in Bonn, Germany. They are all available on my home page. I would like to stress that there are several viewpoints on this vibrant subject held by several other mathematicians and physicists which are equally if not more valid. For a good example, see the review by Yuji Tachikawa, available on his home page.

Looking further to the future, we should not forget that the very existence of the $(2,0)$-theory is but a corollary of the existence of string theory. 
Work on the fundamental principles underlying string theory has noticeably waned-it seems the community is currently gathering more "data" in the form of examples and solid mathematical truths-but ultimately physical mathematics must return to this grand question.

Finally, I would like to comment on physical mathematics more broadly since the very purpose of the Leonard Eisenbud Prize is to encourage work "that brings mathematics and physics closer together." I think the emergent and very lively field of physical mathematics fits this criterion brilliantly. The use of this term in contrast to the more traditional "mathematical physics" by myself and others is not meant to detract from the magnificent subject of mathematical physics but rather to delineate a smaller subfield characterized by a very distinctive set of questions, goals, and techniques. The questions and goals are often motivated, on the physics side, by quantum gravity, string theory, and supersymmetry, and, on the mathematics side, often involve deep relations to topology, geometry, and even analytic number theory in addition to the more traditional relations of physics to algebra, group theory, and analysis. This is a subject which has not been without its critics. Perhaps the most forceful criticism is that of Arthur Jaffe and Frank Quinn. ${ }^{1}$ While these criticisms were very ably answered by Michael Atiyah et al. $^{2}$ and William Thurston, ${ }^{3}$ the issues raised by Jaffe and Quinn are not without merit and we would do well not to forget them. Nevertheless, given the wide spectrum of astonishing results achieved in physical mathematics in the period since this debate erupted, the overwhelming preponderance of evidence is that the subject has great depth and validity. It is likely to remain an important beacon for progress in mathematics for some time to come.

\section{About the Prize}

The Eisenbud Prize was established in 2006 in memory of the mathematical physicist Leonard Eisenbud (1913-2004) by his son and daughterin-law, David and Monika Eisenbud. Leonard Eisenbud, who was a student of Eugene Wigner, was particularly known for the book Nuclear Structure (1958), which he coauthored with Wigner. A friend of Paul Erdós, he once threatened to write a dictionary of "English to Erdős and Erdős to English". He was one of the founders of the Physics Department at the State University of New York, Stony Brook, where he taught from 1957 until his retirement in 1983. His son David was president of

\footnotetext{
${ }^{1}$ Bull. Amer. Math. Soc. (N.S.) 29 (1993) no. 1, 1-13; arXiv:math/9307227 [math.HO].

${ }^{2}$ Bull. Amer. Math. Soc. (N.S.) 30 (1994) no. 2, 178-207; arXiv:math/9404229 [math.HO].

${ }^{3}$ Bull. Amer. Math. Soc. (N.S.) 30 (1994) no. 2, 161-177; arXiv:math/9404236 [math.HO].
}

the AMS during 2003-2004. The Eisenbud Prize for Mathematics and Physics honors a work or group of works that brings the two fields closer together. Thus, for example, the prize might be given for a contribution to mathematics inspired by modern developments in physics or for the development of a physical theory exploiting modern mathematics in a novel way. The US $\$ 5,000$ prize will be awarded every three years for a work published in the preceding six years.

The Eisenbud Prize is awarded by the AMS Council acting on the recommendation of a selection committee. For the 2014 prize, the members of the selection committee were Elliott H. Lieb, Cumrun Vafa, and Eric G. Zaslow.

Previous recipients of the Eisenbud Prize are Hirosi Ooguri, Andrew Strominger, and Cumrun Vafa (2008), and Herbert Spohn (2011).

-Elaine Kehoe 